Juvenal Soares Dias-da-Costa 1 Denise Petrucci Gigante ${ }^{2}$ Ana Maria Baptista Menezes 3 Maria Teresa Anselmo Olinto 4 Silvia Macedo 3 Marcelo Alexandre Pinto de Britto 1 Sandra Costa Fuchs 5

\section{Uso de métodos anticoncepcionais e adequação de contraceptivos hormonais orais na cidade de Pelotas, Rio Grande do Sul, Brasil: 1992 e 1999}

\author{
Contraceptive methods and adequacy \\ of oral contraceptive use in the city of Pelotas, \\ Rio Grande do Sul, Brazil: 1992-1999
}

1 Departamento de Medicina Social, Faculdade de Medicina, Universidade Federal de Pelotas. Av. Duque de Caxias 250, Pelotas, RS 96030-002, Brasil. jsdc@ufpel.tche.br

2 Faculdade de Nutrição, Universidade Federal de Pelotas.

Campus Universitário $s / n$, C. P. 354, Pelotas, RS 96010-900, Brasil.

3 Departamento de Clínica Médica, Faculdade de Medicina, Universidade Federal de Pelotas. Av. Duque de Caxias 250, Pelotas, RS 96030-002, Brasil. ${ }^{4}$ Centro de Ciências da Saúde, Universidade do Vale do Rio dos Sinos. Av. Unisinos 950 , São Leopoldo, RS 93022-000, Brasil. 5 Departamento de Medicina Social, Faculdade de Medicina, Universidade Federal do Rio Grande do Sul. Rua Ramiro Barcelos 2600, sala 415, Porto Alegre, RS 90035-003, Brasil.

\begin{abstract}
A cross-sectional study was conducted to assess contraceptive methods and the adequacy of oral contraceptive use by women aged 20 to 49 years in the city of Pelotas. The results were compared with another cross-sectional study performed in 1992. A sample was randomly selected, including 766 women aged 20 to 49 years. Some 495 of the sample (64.6\%) used a contraceptive method, in the following order: oral contraceptives (55.4\%), surgical sterilization (22.2\%), condoms (10,5\%), and IUD (7.7\%). Among users of oral contraceptives, 62 (22.2\%) had some contraindication. Incorrect use of contraceptive methods was associated with age but not with socioeconomic status. As compared to the previous study, there was a reduction in the use of oral contraceptives. Meanwhile, other methods such as surgical sterilization, condoms, and IUD were used more frequently than in 1992.
\end{abstract}

Key words Contraception; Family Planning; Cross-Sectional Studies

Resumo Realizou-se um estudo transversal de base populacional, com o objetivo de verificar a utilização de métodos contraceptivos e adequação do emprego de anticoncepcionais orais pelas mulheres de 20 a 49 anos, residentes na zona urbana da cidade de Pelotas. Os resultados foram comparados com outro estudo transversal de base populacional, realizado em 1992. Utilizou-se amostragem por conglomerados, incluindo-se 766 mulheres com idade entre 20 e 49 anos. Do total de mulheres, 495 (64,6\%) utilizavam algum método contraceptivo, sendo mais freqüentemente empregados: anticoncepcionais orais (55,4\%), ligadura tubária (22,2\%), preservativo (10,5\%) e dispositivo intra-uterino (7,7\%). Entre as usuárias de anticoncepcionais orais, verificou-se que 22,2\% apresentavam contra-indicação para seu emprego. O uso incorreto associou-se significativamente à idade, mas foi semelhante entre as classes sociais. Comparando os resultados com aqueles do estudo anterior, destaca-se a redução no emprego de anticoncepcionais orais, e maior freqüência de ligaduras, uso de preservativos e dispositivos intra-uterinos.

Palavras-chave Anticoncepção; Planejamento Familiar; Estudos Transversais 


\section{Introdução}

A Organização Mundial da Saúde (OMS) reconhece que o planejamento familiar é uma intervenção com grande potencial de impacto na saúde das populações, desde que se propicie a disponibilidade dos métodos e seu emprego adequado (WHO, 2000).

Através do Programa de Assistência Integral à Saúde da Mulher (PAISM), o Estado brasileiro assumiu uma posição formulando atividades em relação aos direitos reprodutivos de sua população (MS, 1984). Ainda que o PAISM não tenha sido instalado e não seja efetivo em todo o território nacional, é inegável seu significado do ponto de vista de estratégia programática e de paradigma assistencial para o contingente populacional a que está destinado (Osis, 1998).

Pelotas é uma cidade de aproximadamente 300.000 habitantes, que dispõe de uma extensa rede de cuidados primários de saúde. O planejamento familiar integra o conjunto de atividades prioritárias, desenvolvidas pelas unidades sanitárias que compõem o sistema local (SMS, 1996). Desde 1989, a Secretaria Municipal de Saúde tem adotado e seguido as normas do PAISM.

Reconhecidamente, os estudos transversais podem ser utilizados em saúde pública tanto para estabelecer a imagem inicial de situações sanitárias, como para avaliar a efetividade de políticas e ações desenvolvidas (Andrade \& Zicker, 1997).

Em 1992, realizou-se um estudo transversal de base populacional nos habitantes adultos da cidade de Pelotas, que investigou o uso de serviços de saúde (Dias-da-Costa \& Facchini, 1997). Um dos principais resultados foi a descrição do consumo de métodos contraceptivos e adequação do uso de anticoncepcionais orais (Dias-da-Costa et al., 1996).

Assim, em 1999, realizou-se um estudo transversal de base populacional, com o objetivo de verificar a utilização de métodos contraceptivos e adequação do consumo de anticoncepcionais orais pelas mulheres de 20 a 49 anos, residentes na zona urbana da cidade de Pelotas. Os resultados do estudo atual foram comparados com aqueles obtidos em 1992 (Dias-da-Costa et al., 1996), uma vez que ambos seguiram a mesma metodologia, a fim de caracterizar modificações no emprego de métodos anticoncepcionais e na adequação do uso de anticoncepcionais orais.

\section{Materiais e métodos}

Realizou-se um estudo transversal de base populacional, envolvendo diversos aspectos relacionados à saúde da população adulta, de 20 a 69 anos, residente na zona urbana da cidade de Pelotas, Rio Grande do Sul. O trabalho de campo foi realizado entre dezembro de 1999 e abril de 2000.

O estudo atual serve de parâmetro de comparação entre possíveis mudanças ocorridas na política local de planejamento familiar, uma vez que pesquisa com a mesma metodologia e população foi realizada em 1992.

No presente estudo, o tamanho da amostra foi estimado através de diversas prevalências exploradas pelo Projeto Saúde dos Adultos na cidade de Pelotas. Para o cálculo, utilizou-se um poder de $80 \%$, um erro alfa de 0,05 para exposições variando entre $25 \%$ e $75 \%$, com uma razão de prevalência de 1,6. Acrescentou-se ao tamanho da amostra $10 \%$ pela possibilidade de recusas e $15 \%$ para controle de fatores de confusão, o que resultou em uma amostra de 1.800 indivíduos. Dos 281 setores censitários da cidade, quarenta foram sorteados aleatoriamente. Em cada setor foram visitados trinta domicílios, totalizando 1.200 famílias, onde se esperava encontrar 1,5 pessoas da faixa etária do estudo. A partir de um quarteirão previamente sorteado, escolheu-se, também de maneira aleatória, o ponto de partida e a partir do primeiro domicílio selecionado, sistematicamente, saltavam-se duas casas para então escolher a próxima.

Ao final do trabalho de campo, encontrouse $1.145(95,4 \%)$ famílias. Destas, 57 (4,7\%) eram constituídas por indivíduos fora da faixa etária prevista no estudo. Portanto, 55 (4,5\%) famílias foram classificadas como perdas ou recusas.

Foram encontradas 2.177 pessoas de 20 a 69 nos domicílios visitados, porém 1.968 indivíduos foram entrevistados. Dessa forma, as perdas e recusas individuais atingiram 9,6\%.

Do total da amostra encontrou-se 766 $(38,9 \%)$ mulheres entre 20 e 49 anos que foram incluídas no presente estudo. Essa amostra é suficiente para detectar uma prevalência de uso de métodos contraceptivos de cerca de $65 \%$, com um erro aceitável de quatro pontos porcentuais e nível de confiança de $95 \%$.

Aplicou-se um questionário padronizado e pré-codificado a todas as mulheres incluídas na pesquisa. Os entrevistadores foram acadêmicos da Universidade Federal de Pelotas (UFPel), os quais submeteram-se a programa de treinamento e estudo piloto, e desconheciam os ob- 
jetivos do estudo de adequação do consumo de anticoncepcionais orais, desta forma evitandose viés de informação.

Coletou-se informações quanto ao tipo de método contraceptivo utilizado; idade das mulheres; classe social (classificação de Bronfman e Associação Brasileira dos Institutos de Pesquisa de Mercado - ABIPEME); renda familiar per capita em salários mínimos; escolaridade (em anos de estudo completos); número médio de consultas médicas durante o ano e no último mês; acesso a plano de saúde; hipertensão arterial sistêmica referida; diabetes mellitus referido e tabagismo. As variáveis foram coletadas com o mesmo enunciado da investigação realizada em 1992, garantindo-se reprodutibilidade.

Utilizou-se duas classificações de classe social: a de Bronfman (Bronfman et al., 1988) e da ABIPEME (Rutter, 1988). Em função do baixo número de indivíduos encontrados nos extratos populacionais mais extremos da distribuição, para fins de análise estatística, agrupou-se algumas categorias.

Considerou-se inadequado o uso de anticoncepcionais orais quando a usuária referia ser portadora de hipertensão arterial sistêmica, diabetes mellitus, ou era fumante e apresentava idade igual ou maior do que 35 anos (Hatcher et al., 1999; MacKay, 1999; Moore, 1994; Rocha et al., 1996).

Realizou-se controle de qualidade para garantir a confiabilidade das informações, a partir da aplicação de questionários simplificados, em $10 \%$ da amostra estudada. Empregou-se o programa Epi Info, versão 6.0 (Dean et al., 1994), para criar o banco de dados, e os questionários foram digitados em duplicata. Criou-se um programa para checagem de consistência das respostas. Realizou-se a análise dos dados no programa SPSS, versão 8.0 (SPSS Incorporation, 1997), comparando-se as proporções de uso inadequado de anticoncepcionais orais em relação às variáveis em estudo. As razões de prevalências foram estimadas com os respectivos intervalos de confiança de 95\%. A diferença entre as médias de consultas médicas durante um ano e no último mês, foi verificada através do teste de Mann-Whitney, uma vez que a distribuição das variáveis era assimétrica. As prevalências de consumo de anticoncepcionais orais inadequados nos dois estudos, foram comparadas através dos intervalos de confiança de duas proporções de grupos independentes (Altman, 1997).

O Projeto de Pesquisa foi aprovado pelo Comitê de Ética e Pesquisa da Faculdade de Medicina da UFPel.

\section{Resultados}

Entre as 766 mulheres encontradas na faixa etária de 20 a 49 anos, $495(64,6 \%)$ utilizavam algum método contraceptivo. Entre as usuárias de métodos contraceptivos, encontraram-se $274(55,4 \%)$ mulheres utilizando anticoncepcionais orais, $109(22,2 \%)$ com ligadura tubária, $52(10,5 \%)$ usuárias de preservativos e 38 $(7,7 \%)$ com dispositivos intra-uterinos. Observou-se que cinco $(1,0 \%)$ mulheres usavam uma combinação de anticoncepcional oral e preservativos (Tabela 1).

Quanto ao estudo de 1992, 455 (65,7\%) mulheres utilizavam método contraceptivo. Os métodos mais utilizados foram anticoncepcionais orais $(66,5 \%)$, ligadura tubária $(18,4 \%)$, dispositivos intra-uterinos $(4,3 \%)$ e preservativos $(4,0 \%)$. Verificou-se que os intervalos de confiança para as diferenças entre as proporções, não englobaram o valor unitário nas comparações entre os anticoncepcionais orais (IC 95\%: 12,2-2,2), dispositivos intra-uterinos (IC 95\%: 4,1-0,1) e preservativos (IC 95\%: 6,0-2,0). Contudo, os intervalos de confiança da diferença entre as proporções da realização de ligadura tubária englobaram o valor unitário.

Entre as 279 usuárias de anticoncepcionais orais, 22,2\% apresentavam alguma contra-indicação para seu consumo. Entre as contra-indicações, destaca-se que trinta $(10,8 \%)$ mulheres fumavam e tinham idade igual ou maior do que 35 anos, vinte $(7,2 \%)$ apresentavam hipertensão arterial sistêmica, seis $(2,2 \%)$ tinham uma combinação das duas anteriores, três $(1,1 \%)$ eram portadoras de diabetes mellitus e três $(1,1 \%)$ referiram diabetes e hipertensão (Tabela 2).

Tabela

Distribuição dos métodos contraceptivos nas mulheres de 20 a 49 anos. Pelotas, Rio Grande do Sul, 1999 e 1992.

\begin{tabular}{lrrrr}
\hline Variáveis & \multicolumn{2}{c}{1999} & \multicolumn{2}{c}{1992} \\
& $n$ & $\%$ & $n$ & $\%$ \\
\hline Anticoncepcional oral & 274 & 55,4 & 296 & 66,5 \\
Ligadura tubária & 109 & 22,0 & 82 & 18,4 \\
Preservativo & 52 & 10,5 & 18 & 4,0 \\
Dispositivo intra-uterino & 38 & 7,7 & 19 & 4,3 \\
Tabela & 11 & 2,2 & 11 & 2,5 \\
Anticoncepcional oral + preservativo & 5 & 1,0 & - & - \\
Anticoncepcional injetável & 3 & 0,6 & 8 & 1,8 \\
Coito interrompido & 2 & 0,4 & 1 & 0,2 \\
Diafragma & 1 & 0,2 & 1 & 0,2 \\
Vasectomia & - & - & 3 & 0,7 \\
\hline
\end{tabular}


Em relação à adequação do consumo de anticoncepcionais orais, no presente estudo constatou-se que $62(22,2 \%)$ mulheres apresentavam pelo menos uma contra-indicação para sua utilização. Esse porcentual foi maior do que o encontrado em 1992 (18,6\%). O estudo atual adicionou como contra-indicação a referência de diabetes mellitus. Mesmo retirando-se da análise as pacientes que referiram apresentar diabetes, o percentual manteve-se superior ao encontrado em 1992. Contudo, a análise através dos intervalos de confiança de 95\% da diferença entre as proporções não foi significativo (IC 95\%: 6,5-6,6).

Explorou-se a associação de uso inadequado de anticoncepcionais orais com as variáveis sócio-econômicas, e se possuía plano de saúde. Contudo, os intervalos de confiança das razões de prevalência englobaram a unidade e os testes estatísticos não foram significativos. Verificou-se que a prevalência de mulheres que apresentavam uso inadequado, aumentava à medida que se elevavam os extratos etários utilizados no estudo (Tabela 3).

Não foram observadas diferenças entre as médias de consultas durante um ano e no último mês, entre as mulheres com uso adequado e inadequado de anticoncepcionais orais. As médias de consultas no último ano foram de 3,5 e 3,4, respectivamente, enquanto que as médias no último mês atingiram a 0,4 e 0,3.

\section{Discussão}

Uma das vantagens dos estudos populacionais é a de que a partir de seus achados, podem-se realizar inferências e estimativas para toda a população.

A estrutura da amostra por faixa etária foi semelhante à projetada pela Fundação Institu-

Tabela 2

Distribuição das contra-indicações para emprego de anticoncepcionais orais na população de Pelotas. Rio Grande do Sul, 1999.

\begin{tabular}{lrc}
\hline \multirow{2}{*}{ Contra-indicação } & \multicolumn{2}{c}{1999} \\
& $n$ & \multicolumn{2}{c}{$\%$} \\
\hline Sem contra-indicação & 217 & 77,8 \\
Fumo e idade $\geq 35$ anos & 30 & 10,8 \\
Hipertensão arterial sistêmica & 20 & 7,2 \\
Fumo e idade $\geq 35$ anos e hipertensão & 6 & 2,2 \\
Diabetes mellitus & 3 & 1,1 \\
Diabetes mellitus e hipertensão & 3 & 1,1 \\
\hline
\end{tabular}

to Brasileiro de Geografia e Estatística (IBGE) para a zona urbana da cidade de Pelotas, o que confirmou a representatividade do estudo e demonstrou que não houve viés de seleção.

Na discussão do artigo publicado em 1996, advertiu-se que em função do elevado número de mulheres consumindo anticoncepcionais orais $(66,5 \%)$ e da baixa freqüência de utilização de outros métodos, evidenciava-se que havia basicamente apenas um meio de contracepção disponível para a população feminina em 1992 (Dias-da-Costa et al., 1996).

A pouca utilização de outros métodos contraceptivos, além da ligadura tubária e dos anticoncepcionais orais, também tem sido detectada e discutida por outros autores (Kalckmann et al., 1997; Villela \& Barbosa, 1996).

As informações coletadas em Pelotas, no período de 1999 e 2000, mostraram uma alteração dessa realidade, uma vez que os principais métodos utilizados foram anticoncepcional oral $(56,4 \%)$, ligadura tubária $(22,0 \%)$, preservativos $(10,5 \%)$ e dispositivos intra-uterinos $(7,7 \%)$. A partir do novo modelo de consumo, percebeuse que houve uma diminuição de $15,0 \%$ quanto ao uso de anticoncepcionais orais. Porém, quanto à utilização de outros métodos, verificou-se um crescimento de $19,6 \%$ em relação à ligadura tubária, 79,1\% de dispositivos intrauterinos e 162,5\% de preservativos. Esses achados podem, aparentemente, sugerir uma ampliação na disponibilidade dos outros métodos, a partir da implantação do PAISM na cidade de Pelotas. De fato, a prevalência da utilização de dispositivos intra-uterinos foi um achado surpreendente e por tratar-se de um método eficaz, ressaltou-se como positivo o aumento de seu uso. O grande aumento no consumo de preservativos pode ser explicado como reflexo da epidemia de AIDS, embora apenas cinco mulheres utilizassem a combinação de anticoncepcional oral e preservativos. Contudo, os resultados seguem revelando uma limitação na oferta de outros métodos; nenhuma mulher, por exemplo, mencionou como método vasectomia do marido.

Ainda que perdure uma elevada concentração no consumo de anticoncepcionais orais entre as mulheres de Pelotas, a situação difere da encontrada no Brasil como um todo, onde a esterilização feminina é o método mais prevalente $(40,1 \%$ das mulheres unidas de 15 a 49 anos), seguido do uso de anticoncepcionais orais $(20,7 \%)$ (BEMFAM/Macro International, 1997). Outro estudo de base populacional, realizado na zona sul do Município de São Paulo, incluindo mulheres de 10 a 49 anos, mostrou como métodos mais prevalentes a esterilização 


\begin{tabular}{|c|c|c|c|}
\hline Variável & $\begin{array}{c}\text { Uso inadequado } \\
n(\%)\end{array}$ & Razão de prevalência & Intervalo de confiança \\
\hline \multicolumn{4}{|l|}{ Classe social (ABIPEME) } \\
\hline Classes A e B & $12(15,4)$ & 1,00 & \\
\hline Classe C & $26(23,6)$ & 1,54 & $0,83-2,85$ \\
\hline Classes D e E & $23(25,6)$ & 1,66 & $0,89-3,11$ \\
\hline \multicolumn{4}{|l|}{ Classe social (Bronfman) } \\
\hline Burguesia e nova pequena burguesia & $6(13,6)$ & 1,00 & \\
\hline Pequena burguesia tradicional & $14(27,5)$ & 2,01 & $0,85-4,79$ \\
\hline Proletariado não típico & $27(23,1)$ & 1,51 & $0,75-3,82$ \\
\hline Proletariado & $27(23,1)$ & 1,51 & $0,56-4,08$ \\
\hline Subproletariado & $7(23,3)$ & 1,71 & $0,64-4,59$ \\
\hline \multicolumn{4}{|l|}{ Renda familiar em salários mínimos } \\
\hline 3,01 ou mais & $9(14,1)$ & 1,00 & \\
\hline 1,01 a 3,0 & $34(26,8)$ & 1,90 & $0,97-3,72$ \\
\hline$<1,01$ & $19(21,6)$ & 1,54 & $0,74-3,17$ \\
\hline \multicolumn{4}{|l|}{ Escolaridade (anos) } \\
\hline Mais de 15 & $6(19,4)$ & 1,00 & \\
\hline 12 a 15 & $12(14,1)$ & 0,73 & $0,30-1,78$ \\
\hline 8 a 11 & $16(25,8)$ & 1,33 & $0,58-3,07$ \\
\hline 5 a 7 & $13(22,0)$ & 1,14 & $0,48-2,70$ \\
\hline 0 a 4 & $15(33,7)$ & 1,85 & $0,81-4,25$ \\
\hline \multicolumn{4}{|l|}{ Idade (anos) } \\
\hline $20-29$ & $11(8,6)$ & 1,00 & \\
\hline $30-39$ & $30(30,0)$ & 3,53 & $1,86-6,68$ \\
\hline $40-49$ & $21(40,4)$ & 4,70 & $2,44-9,04$ \\
\hline \multicolumn{4}{|l|}{ Plano de saúde } \\
\hline $\operatorname{Sim}$ & $22(18,3)$ & 1,00 & \\
\hline Não & $40(25,2)$ & 1,37 & $0,86-2,18$ \\
\hline
\end{tabular}

ABIPEME = Associação Brasileira dos Institutos de Pesquisa de Mercado.

feminina $(42,9 \%)$ e anticoncepcionais orais (35,3\%) (Schor et al., 2000).

Ainda que o uso de anticoncepcionais orais seja seguro, o porcentual encontrado deve ser considerado elevado, indicando problemas na qualidade da assistência médica ambulatorial oferecida na cidade de Pelotas. Deve-se salientar que apesar das mulheres terem consultado com médico numa freqüência razoável (em média 3,4 vezes durante o último ano), a persistência do consumo na presença de contra-indicações sugere falta de integralidade na atenção. Além disso, sabe-se que a principal causa de mortalidade em Pelotas, no Rio Grande do Sul, são as doenças do aparelho cardiocirculatório (SSMA, 1996). O enfrentamento desse problema incorre na prevenção dos fatores de risco, entre os quais o uso inadequado de anticoncepcionais orais (WHO, 1993).

O fato das mulheres na faixa etária acima de trinta anos, apresentarem maior consumo inadequado de anticoncepcionais orais era esperado, uma vez que as contra-indicações especificadas no atual estudo são mais prevalentes com o aumento da idade (Malerbi \& Franco, 1992; Piccini \& Victora, 1994).

Comparou-se a inadequação do uso de anticoncepcionais orais com as duas classificações de classe social e com a renda familiar com o intuito de detectar iniqüidade na atenção às mulheres. Assim como ocorreu em 1992, não encontrou-se diferenças no consumo ina- 
dequado de anticoncepcionais entre as classes sociais. Entretanto, uma análise mais detalhada, revelou que nenhuma mulher da burguesia (classificação de Bronfman), apenas uma com renda maior do que 10,1 salários mínimos (renda familiar per capita em salários mínimos) e somente três mulheres da classe A (classificação da ABIPEME), apresentavam uso inadequado de anticoncepcionais orais, sugerindo

\section{Agradecimentos}

Projeto de Pesquisa financiado pela Fundação de Amparo à Pesquisa do Estado do Rio Grande do Sul (FAPERGS).

\section{Referências}

ALTMAN, D. G., 1997. Practical Statistics for Medical Research. London: Chapman \& Hall.

ANDRADE, A. L. S. S. \& ZICKER, F., 1997. Estudos de prevalência. In: Métodos de Investigação Epidemiológica em Doenças Transmissíveis: Volume I (A. L. S. S. Andrade \& F. Zicker, org.), pp. 33-42, Brasília: Organização Pan-Americana da Saúde/ Fundação Nacional de Saúde.

BEMFAM (Sociedade Civil Bem-Estar Familiar no Brasil)/MACRO INTERNATIONAL, 1997. Pesquisa Nacional Sobre Demografia e Saúde, 1996. Rio de Janeiro: BEMFAM/Macro International.

BRONFMAN, M.; LOMBARDI, C.; FACCHINI, L. A.; VICTORA, C. G.; BARROS, F. C.; BÉRIA, J. U. \& TEIXEIRA, A. M. B., 1988. Operacionalização do conceito de classe social em estudos epidemiológicos. Revista de Saúde Pública, 22:253-265.

DEAN, A. G.; DEAN, J. A.; COULOMBIER, D.; BRENDEL, K. A.; SMITH, D. C.; BURTON, A. H.; DICKER, R. C.; SULLIVAN, K.; FAGAN, R. F. \& ARNER, T. G., 1994. Epi Info, Version 6: A Word Processing, Database and Statistics Program for Epidemiology on Microcomputers. Atlanta: Centers for Disease Control and Prevention. falta de poder no estudo para detectar diferenças entre as classes. Assim, poderia-se supor e apontar, com base na leitura dos números absolutos nas categorias de classe social, que correspondiam aos extratos mais elevados, a possível existência de uma indesejável iniqüidade na atenção à saúde reprodutiva das mulheres de Pelotas.
DIAS-DA-COSTA, J. S.; D’ELIA, P. B. \& MOREIRA, M. R., 1996. Prevalência de uso de métodos contraceptivos e adequação do uso de anticoncepcionais orais na cidade de Pelotas, Rio Grande do Sul, Brasil. Cadernos de Saúde Pública, 12:141-147.

DIAS-DA-COSTA, J. S. \& FACCHINI, L. A., 1997. Utilização de serviços ambulatoriais em Pelotas: Onde a população consulta? Com que freqüência? Revista de Saúde Pública, 31:360-369.

HATCHER, R. A.; RINEHART, W.; BLACKBURN, R.; GELLER, J. S. \& SHELTON, J. D., 1999. Lo Esencial de la Tecnologia Anticonceptiva. Baltimore: School of Public Health, Johns Hopkins University.

KALCKMANN, S.; LAGO, T. G.; BARBOSA, R. M.; VILLELA, W. \& GOIHMAN, S., 1997. O diafragma como método contraceptivo: A experiência de usuárias de serviços públicos de saúde. Cadernos de Saúde Pública, 13:647-657.

MacKAY, H. T., 1999. Contraception. In: Current Medical \& Treatment 1999 (L. M. Tierney, S. J. McPhee, \& M. A. Papadakis, ed.), pp. 725-729, Stamford: Appleton \& Lange.

MALERBI, D. \& FRANCO, L. J., 1992. The Brazilian Cooperative Group on the Study of Diabetes Preva- 
lence Multicenter study of the prevalence of diabetes mellitus and impaired glucose tolerance in the urban brazilian population aged 30-69 yr. $D i$ abetes Care, 15:1509-1516.

MS (Ministério da Saúde), 1984. Assistência Integral à Saúde da Mulher: Bases de Ação Programática. Brasília: Centro de Documentação, Ministério da Saúde.

MOORE, J. G., 1994. Anticoncepção e esterilização. In: Fundamentos de Ginecologia e Obstetrícia (N. F. Hacker \& J. G. Moore, org.), pp. 314-323, Porto Alegre: Artes Médicas.

OSIS, M. J. D., 1998. PAISM: Um marco na abordagem da saúde reprodutiva no Brasil. Cadernos de Saúde Pública, 14(Sup. 1):25-32.

PICCINI, R. X. \& VICTORA, C. G., 1994. Hipertensão arterial sistêmica em área urbana no Sul do Brasil: Prevalência e fatores de risco. Revista de Saúde Pública, 28:261-267.

ROCHA, M. L.; LISBOA, K. O. \& GIUGLIANI, E. R. J., 1996. Planejamento familiar. In: Medicina Ambulatorial: Condutas Clínicas em Atenção Primária (B. B. Duncan, M. I. Schimidt \& E. R. J. Giugliani, org.), pp. 175-182, Porto Alegre: Artes Médicas.

RUTTER, M., 1988. Pesquisa de Mercado. São Paulo: Editora Ática.

SCHOR, N.; FERREIRA, A. F.; MACHADO, V. L.; FRANÇA, A. P.; PIROTTA, K. C. M.; ALVARENGA,
A. T. \& SIQUEIRA, A. A. F., 2000. Mulher e anticoncepção: Conhecimento e uso de métodos anticoncepcionais. Cadernos de Saúde Pública, 16:377384.

SSMA (Secretaria da Saúde e do Meio Ambiente), 1996. Estatísticas de Saúde: Mortalidade 1995. Porto Alegre: SSMA.

SMS (Secretaria Municipal de Saúde), 1996. Plano Municipal de Saúde. Pelotas, 1996. Pelotas: Editora Universitária.

SPSS INCORPORATION, 1997. SPSS for Windows. Statistical Package for the Social Sciences. Release 8.0. Chicago: SPSS Inc.

VILLELA, W. \& BARBOSA, R., 1996. Opções contraceptivas e vivências de sexualidade: Comparação entre mulheres esterilizadas e não esterilizadas em região metropolitana do Sudeste do Brasil. Revista de Saúde Pública, 30:452-459.

WHO (World Health Organization), 1993. 1993 Guidelines for the management of mild hypertension: Memoradum from a WHO/ISH meeting. Bulletin of the World Health Organization, 71:503-517.

WHO (World Health Organization), 2000. The World Health Report 2000. Health Systems: Improving Performance. Geneva: WHO.

Recebido em 26 de dezembro de 2000

Versão final reapresentada em 3 de maio de 2001

Aprovado em 9 de julho de 2001 